\title{
A BAYESIAN MODEL UPDATING PROCEDURE FOR DYNAMIC HEALTH MONITORING
}

\author{
Edoardo Patelli ${ }^{1}$, Matteo Broggi ${ }^{2}$, and Pierre Beaurepaire ${ }^{2}$ \\ ${ }^{1}$ Institute of Risk and Uncertainty, University of Liverpool \\ Brodie Tower, L69 3GQ Liverpool, UK \\ e-mail: edoardo.patelli@liverpool.ac.uk \\ ${ }^{2}$ Virtual Engineering Centre, University of Liverpool \\ STFC Daresbury Laboratory, Daresbury Science and Innovation Campus, WA4 4AD Warrington, UK \\ e-mail: \{matteo.broggi,pierre.beaurepaire\}@ liverpool.ac.uk
}

Keywords: Bayesian Model Updating, Transitional Markov Chain Monte Carlo, Fatigue, Crack Detection, Dynamic Excitation, Health Monitoring.

\begin{abstract}
Structures under dynamic excitation can undergo phenomena of crack growth and fracture. For safety reasons, it is of key importance to be able to detect and classify these cracks before the unwarned structural failure. Additionally, the cracks will also change the dynamic behaviour of the structures, impacting their performance.

Here, a Bayesian model updating procedure has been implemented for the crack detection location and length estimation on a numerical model of a spring suspension arm. A highfidelity finite element model has been used to simulate experimental data, by inserting cracks of different extent at different locations and obtaining reference frequency response functions. In the following, a low fidelity parametric model has been used in the Bayesian framework to infer the crack location and length by comparing the dynamic responses. It is shown that the proposed methodology can be successfully adopted as a structural health monitoring tool.
\end{abstract}




\section{INTRODUCTION}

Fatigue is the most dangerous failure mode for mechanical components subject to alternating loads: one or several cracks can be initiated and propagated through the cross section of the structure. Once a critical crack length is exceeded, the structure can catastrophically fail even for stress level much lower than the design stress [1]. In particular, interactions may occur between the structural responses and cracks in components subject to high frequency dynamic excitations, leading to vibration-induced fatigue. In this case, the stress field in the structure is mainly determined by the high frequency resonance modes, leading to very fast cycles of loading and accelerated fatigue crack growth. Hence the service life of the structure may be considerably reduced.

Several strategies are possible to avoid fracture; for instance, non-destructive inspections may be performed at predetermined time intervals in order to detect the cracks; however failure can happen between inspections [2]. Alternatively, a continuous monitoring of the dynamic response of the structure can allow for real-time crack detection and for a timely intervention with maintenance procedures [3]. Repair actions are taken in case the monitoring procedure successfully identifies a crack which jeopardizes the structure.

In both cases, the procedure may fail in identifying a crack, leading to fatigue failure. Thus, efficient crack detection before fracture occurs is required in order to avoid the loss of the structure, and more importantly to mitigate the consequences that such loss could cause, both from an economical and safety point of view. Once the crack is successfully detected, corrective maintenance or substitution of the damaged component can be performed.

Thanks to the advancements in the field of computational mechanics, new detection techniques could be developed to assist in the monitoring of the health of the structures. These numerical techniques allow for a synthetic analysis of experimental and sensor data. More specifically, these techniques modify some specific parameters in a numerical model to ensure a good agreement with the data, leading to a so-called inverse problem. A computational framework well fitted for the solution of such inverse problems is the model updating [4].

In this paper, an efficient numerical framework, based on a Bayesian model updating procedure [5], is implemented for the identification of cracks within structures under dynamic excitation. The influence of the cracks on the mechanical behaviour is quantified using the Frequency Response Functions (FRF) at a specific locations. A suspension arm, as normally used by automotive industry, has been analysed. Two finite elements models have been used: one high-fidelity model to simulate experimental data of the arm under dynamic excitation (i.e., the reference model). Cracks of random dimension and positions determined by stress intensity are introduced in the simulated experimental suspension arm.

The second low-fidelity model is used in the Bayesian model updating procedure. The updating procedure adjusts the length of cracks inserted at candidate crack locations in the lowfidelity model in order to minimize the difference between the frequency response function of this model and the reference frequency response function. This allows reconstructing efficiently the crack pattern of the reference model.

Particular attention is given to the efficiency of the numerical simulation. As a matter of fact, a high number of model evaluation is required, thus a strategy for the parallelization of the simulations as provided by the general purpose software COSSAN-X [6] is employed.

The paper is structured as follows: Section 2 deals with the modelling of fracture in a Finite Element framework. Section 3 outlines the main concept of Bayesian model updating and the efficient simulation algorithm employed in the particular case of structure with cracks under dy- 
namic excitation. A numerical example will be introduced in Section 4, and the crack detection routine will be tested in different configurations. Finally, some conclusions and final remarks will be pointed out in Section 5.

\section{MODELLING OF FRACTURE MECHANICS}

The mechanical behaviour of structures may be modified by the presence of cracks. The effective cross section of the component is reduced, which causes a reduction of the stiffness. Moreover, the stress field is also modified in the vicinity of a crack.

Specific methods allow modelling efficiently the mechanical behaviour of structures containing cracks. The extended finite elements method (XFEM) [7, 8] has received considerable attention over the past few years. It consists of using a particular element formulation in the vicinity of a crack. Additional degrees of freedom and shape functions are used to describe the displacements within an element affected by a crack and such elements are said to be enriched. The displacement field in these elements is then expressed using a combination of the standard and of the shape functions devoted to enrichment.

In case an element is crossed by a crack, the additional shape function consists of a Heaviside function centered on the crack. The discontinuity of this function accounts for discontinuity of the displacements between the two lips of the crack.

In case an element includes the crack tip, the corresponding nodes of the finite element model are enriched with specific shape functions. These functions correspond to the asymptotic displacement field at the vicinity of a crack tip, which can be determined analytically (see [7] for more details about the enrichment of the tip elements). This allows capturing efficiently the displacement and strain fields near the crack tip, without excessive refinement of the mesh.

Details on the implementation of the extended finite element method may be found e.g. in $[7,9,8,10]$.

However, mesh refinement in the vicinity of the crack tip may be necessary when the extended finite elements method is used, in spite of the enrichment of the nodes at the crack tip [11]. Nevertheless, the mesh does not have to be compatible with the crack, which considerably simplifies the re-meshing.

In case the behaviour of a cracked structure under dynamic excitation needs to be determined, the stiffness matrix may be computed using the XFEM, as stated above. The mass matrix is not modified by the presence of cracks, and no special action needs to be taken. The problem is subsequently solved using the standard procedure for linear dynamics: the modes and frequency of vibration are determined by solving the eigen-value problem associated with the mass and stiffness matrices; and the FRFs associated with any pair of DOF of the node of the finite element model are determined.

\section{BAYESIAN MODEL UPDATING FOR CRACK DETECTION}

\subsection{Bayesian updating of structural models}

A Bayesian model updating procedure is based on the well known Bayes theorem [12]. This theorem was first introduced by Thomas Bayes in the 18th century, then was rediscovered and largely redeveloped by Laplace in the 19th century, and finally has been widely accepted and applied only in recent times. Its general formulation is

$$
P(\theta \mid D, I)=\frac{P(D \mid \theta, I) P(\theta \mid I)}{P(D \mid I)}
$$


where $\theta$ represents any hypothesis to be tested, e.g., the value of the model parameters, $D$ is the available data or observations, and $I$ is the background information. Three main terms can be identified in the Bayes theorem:

- $P(\theta \mid I)$ is the prior probability density function (PDF) of the parameters

- $P(\theta \mid D, I)$ is the posterior PDF

- $P(D \mid \theta, I)$ is the likelihood function of the data $\mathrm{D}$.

Finally, the term $P(D \mid I)$ at the denominator is a normalization factor ensuring that the posterior PDF integrates to 1.The theorem introduces a way to update some a priori knowledge on the parameters $\theta$ by using data/observations, conditional to the available information.

Bayes theorem has been successfully applied in the updating of structural models $[13,14]$; in particular the Bayesian structural model updating has been successfully used to update large finite element models using experimental modal data [5].

In a structural model updating framework, the initial knowledge about the unknown adjustable parameters is expressed through the prior PDF. A careful choice of this distribution has to be taken, in order to accurately represent the knowledge available, e.g., from prior expertise. As an example, a proper prior distribution can be a uniform distribution in the case when only a lower and upper bound of the parameter is known, or a Gaussian distribution when the mean and a relative error of the parameter is known.

The likelihood function gives a measure of the agreement between the available experimental data and the corresponding numerical model output. Particular care has to be taken in the definition of the likelihood, and the choice of likelihood depends on the type of data available, e.g., whether the data is a scalar or a vector quantity.

Finally, the posterior distribution expresses the revised knowledge about the parameters, providing information on which parameter ranges are more probable based on the initial knowledge and the experimental data.

\subsection{Transitional Markov-Chain Monte-Carlo}

The Bayesian updating expressed in equation 1 needs the computation of a normalizing factor, that can be very complex to compute and computationally expensive. An efficient stochastic simulation algorithm, called Transitional Markov Chain Monte-Carlo [15], can be used to avoid the computation of this factor. This algorithm allows the generation of samples from the complex shaped unknown posterior distribution through an iterative approach. In this algorithm, $m$ intermediate distributions $P_{i}$ are introduced,

$$
P_{i} \propto P(D \mid \theta, I)^{\beta_{i}} P(\theta \mid I)
$$

where the contribution of the likelihood is scaled down by an exponent $\beta_{i}$, with $0=\beta_{0}<$ $\ldots<\beta_{i}<\ldots<\beta_{m}=1$, thus the first distribution is the prior PDF, and the last is the posterior. These intermediate distributions show a more gradual change in the shape from one step to the next when compared with the shape variation from the prior to the posterior. The value of $\beta_{i}$ is automatically set to allow a more slow variation for the first steps, and a faster variation towards the end. The variation of $\beta$ between two steps is controlled by the value of the coefficient of variation of the intermediate posterior, that is compared to a target $\mathrm{CoV}$ value set as an input of the algorithm. 
In a first step, samples are generated from the prior PDF using direct Monte-Carlo. Then, sample from the $P_{i+1}$ distribution are generated using Markov chains with the MetropolisHasting algorithm [16], starting from selected samples taken from the $P_{i}$ distribution. This step is repeated until the distribution characterized by $\beta_{i}$ is reached. By using the MetropolisHasting algorithm, samples are generated from the posterior PDF without the necessity of ever computing the normalization constant.

\subsection{Model updating for crack detection}

The approach introduced so far has been successfully applied to structural model updating, thus reducing the uncertainties in the numerical model parameters and improving the agreement between the numerical results and experimental data. Within the model updating framework, the cracks' extent and position present in the damaged structure are seen as uncertain model properties.

Knowing that cracks will develop most likely in locations characterized by high concentration of stresses, these candidate positions are inserted in the undamaged model, analysed using XFEM assuming the crack length and shape as random parameters.

In the updating procedure, the prior distribution will use a uniform distribution for the crack parameters, allowing the possibility of crack in any stress concentration point and with any possible physically acceptable length.

Experimental data from the reference structure are available in the form of Frequency Response Functions (FRF). These reference data are compared with the numerical FRF of the numerical model, by computing the root mean squared error (RMSE), in order to include the experimental data information in the likelihood. It is assumed that the prediction error is distributed according to a Gaussian PDF, thus the likelihood can be expressed as

$$
P(D \mid \theta, I) \propto \exp \left(-\frac{1}{2 \delta} \sum_{k=1}^{N_{\text {freq }}}\left(h_{\exp }\left(\omega_{k}\right)-h\left(\omega_{k} ; \theta\right)\right)^{2}\right)
$$

where $h_{\text {exp }}\left(\omega_{k}\right)$ is the experimental FRF, $h\left(\omega_{k} ; \theta\right)$ represents the numerical FRF, and $\delta$ is the variance of the RMSE. After the updating procedure, the posterior will provide a qualitative indication on both crack length and position, i.e., the length of the crack will go towards zero if the crack is not present in the candidate location, and otherwise, it will concentrate around the unknown length.

\section{Numerical Example}

\subsection{Model description}

In this numerical example, the proposed framework is applied to detect cracks in a suspension arm similar to those used in the automotive industry [17]. The structure, shown in Figure 2 can freely rotate along the axis indicated by the dashed line, and the suspension spring and the wheel structure is connected at the location indicated by $\mathrm{S}$. The stress concentration points, and candidate crack locations, are indicated in the figure by the numbers 1 to 6 .

In this example, "simulated" experimental data are generated using a high-fidelity FE model, characterized by a very refined mesh near the crack (Figure $2 b$ ). The software used to construct the model and in the analysis is Code_ASTER [18]. A crack with fixed length is inserted in one of the candidate position, and the reference FRF is computed at the position indicated by "O". Both the FRF in direction $\mathrm{X}$ and $\mathrm{Y}$ are considered, while no FRF is obtained in the direction $\mathrm{Z}$ 


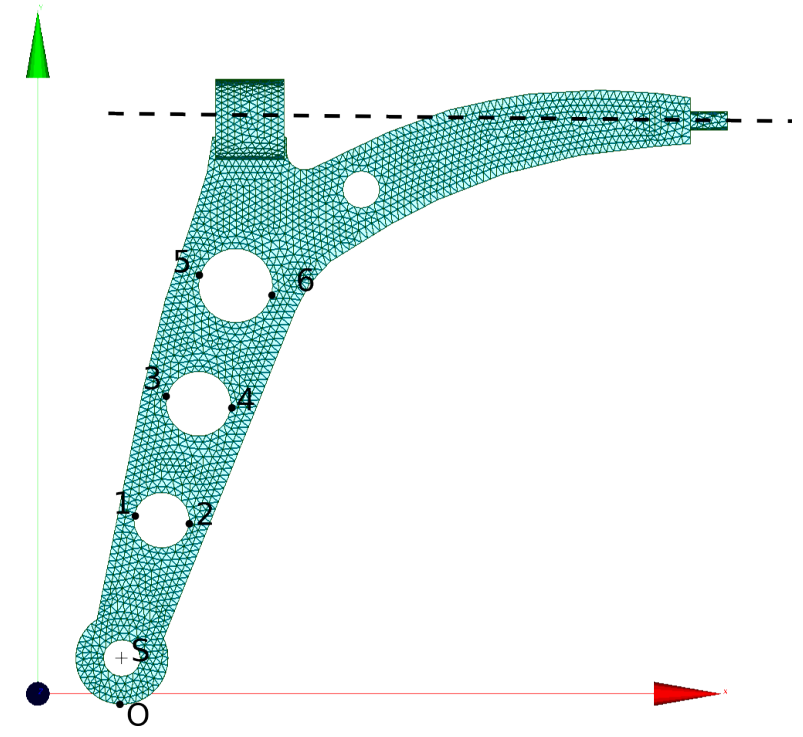

Figure 1: Finite Element model of a suspension arm.

since the structure is not constrained in that direction. Figure 3 shows the inertance FRF in the two directions $\mathrm{X}$ and $\mathrm{Y}$ when a crack with the length of $5 \mathrm{~mm}$ is inserted in the possible positions. A clear difference is shown between the inertance FRF, especially at the high frequencies.

\subsection{Bayesian model updating}

The Bayesian model updating procedure is employed using the low-fidelity FE model, where a more coarse mesh is used. The crack lengths are considered as uncertain parameters, and are modelled using uniformly distributed random variables. Since the crack is physically constrained to not touch the flanges of the arm, a maximum crack length of $5 \mathrm{~mm}$ is assigned to the cracks in position 1 and 2, while the length is limited to $10 \mathrm{~mm}$ for the cracks in positions 3 to 6 . Thus the prior uncertain parameters and their prior distributions are:

$$
\theta=\left\{\begin{array}{c}
l_{1} \\
l_{2} \\
l_{3} \\
l_{4} \\
l_{5} \\
l_{6}
\end{array}\right\}, \quad \theta_{0} \sim\left\{\begin{array}{c}
U(0,5 \mathrm{~mm}) \\
U(0,5 \mathrm{~mm}) \\
U(0,10 \mathrm{~mm}) \\
U(0,10 \mathrm{~mm}) \\
U(0,10 \mathrm{~mm}) \\
U(0,10 \mathrm{~mm})
\end{array}\right\}
$$

The sampled values of the random variables are inserted into the FE model by using the ASCII file injection routine provided by COSSAN-X [6].

The Bayesian updating procedure has been executed with one crack. A reference FRF has been obtained with the high fidelity model with a $5 \mathrm{~mm}$ crack located in position 3 . Then, 1000 samples from the prior distribution have been generated. The low-fidelity FE model is run in parallel on a computer cluster allowing a reduction of the overall computational time. Figure 4 shows the variation of the inertance FRFs of the low-fidelity model with 1600 random values of crack length sampled from the prior distributions.

The FRFs are compared with the reference FRFs from the high-fidelity model, computing the RMSE. In order to speed up the analyses needed for the bayesian updating, a Multi Layer Perceptron Artificial Neural Network has been constructed. The network is constituted by $N_{i n p}=6$ 


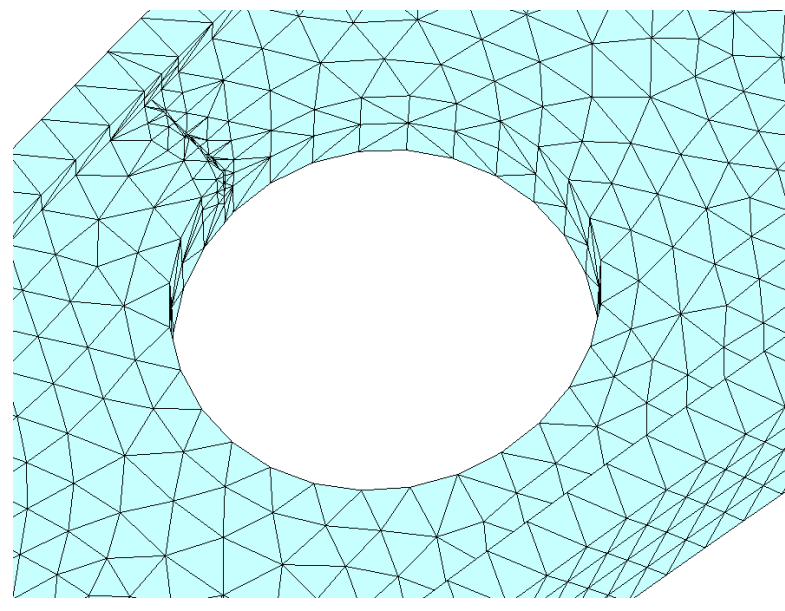

(a) Low fidelity model

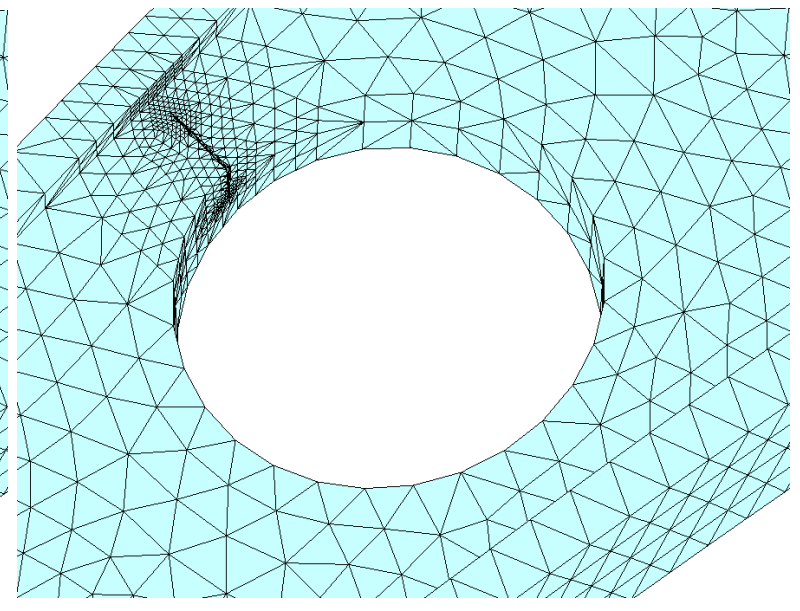

(b) High fidelity model

Figure 2: Low fidelity (a) and high fidelity (b) model of the suspension arm. High mesh refinements near the crack tips are included in the high fidelity model.

inputs, that are the length of the cracks at positions 1 to 6 , and $N_{\text {out }}=2$ outputs, the RMSE computed between the reference FRF and that of the low-fidelity model in bot the $x$ and $y$ direction. An automated training procedure has been implemented such that various network topologies are tested and the best network, characterized by the highest $R^{2}$ value is kept. $R^{2}$ is defined as

$$
R^{2}=1-\frac{\sum_{i=1}^{N_{\text {data }}}\left(h_{i}^{2}-{\hat{h_{i}}}^{2}\right)}{\sum_{i=1}^{N_{\text {data }}}\left(h_{i}^{2}-{\overline{h_{i}}}^{2}\right)},
$$

where $h_{i}$ is the RMSE computed using the real model outputs, $\overline{h_{i}}$ is the average of $h_{i}$, and $\hat{h}_{i}$ is the RMSE predicted by the neural network. The best network is structured with 2 hidden layers, with 13 nodes in the first hidden layer and 11 nodes in the second. The $R^{2}$ is 0.972 for the RMSE of the FRFs in the $x$ direction, and 0.969 for that of the FRFs in the $y$ direction.

\subsection{Discussion of the results}

The results of the updating procedure are shown in Figure 5, where the prior and posterior distributions of the six random crack lengths is shown, while Figure 6 shows the value of the RMSE of the two FRFs using the prior and posterior distributions of the parameters. The procedure successfully updated the model parameters and identified the position of the crack as being position 3 and length $5 \mathrm{~mm}$. The posterior distribution of the crack length clearly remove any possibility of having cracks at positions 1,5 and 6 , since their final PDF is very peaked in the vicinity of zero (see Figures 5a, 5e and 5f).

However, a perfect model updating would have resulted in posterior distributions very peaked around zero for the position 2 and 4 as well, and peaked around 5 for the posterior of the crack length at position 3 as the synthetic numerical simulated data is noise free. This is not the case: instead, some uncertainty is still shown in the posterior distribution for the crack in positions 2 , 3 and 4 . Nevertheless, it can be reasonably assured that the crack is indeed located in position 3 , since the posterior of the crack length at position 3 is the only one having a significant mean value (see Figure 5c). Additionally, the posterior PDF in position 3 is the only PDF not including zero as a possible value of the distribution, as opposed for the distributions relevant to 

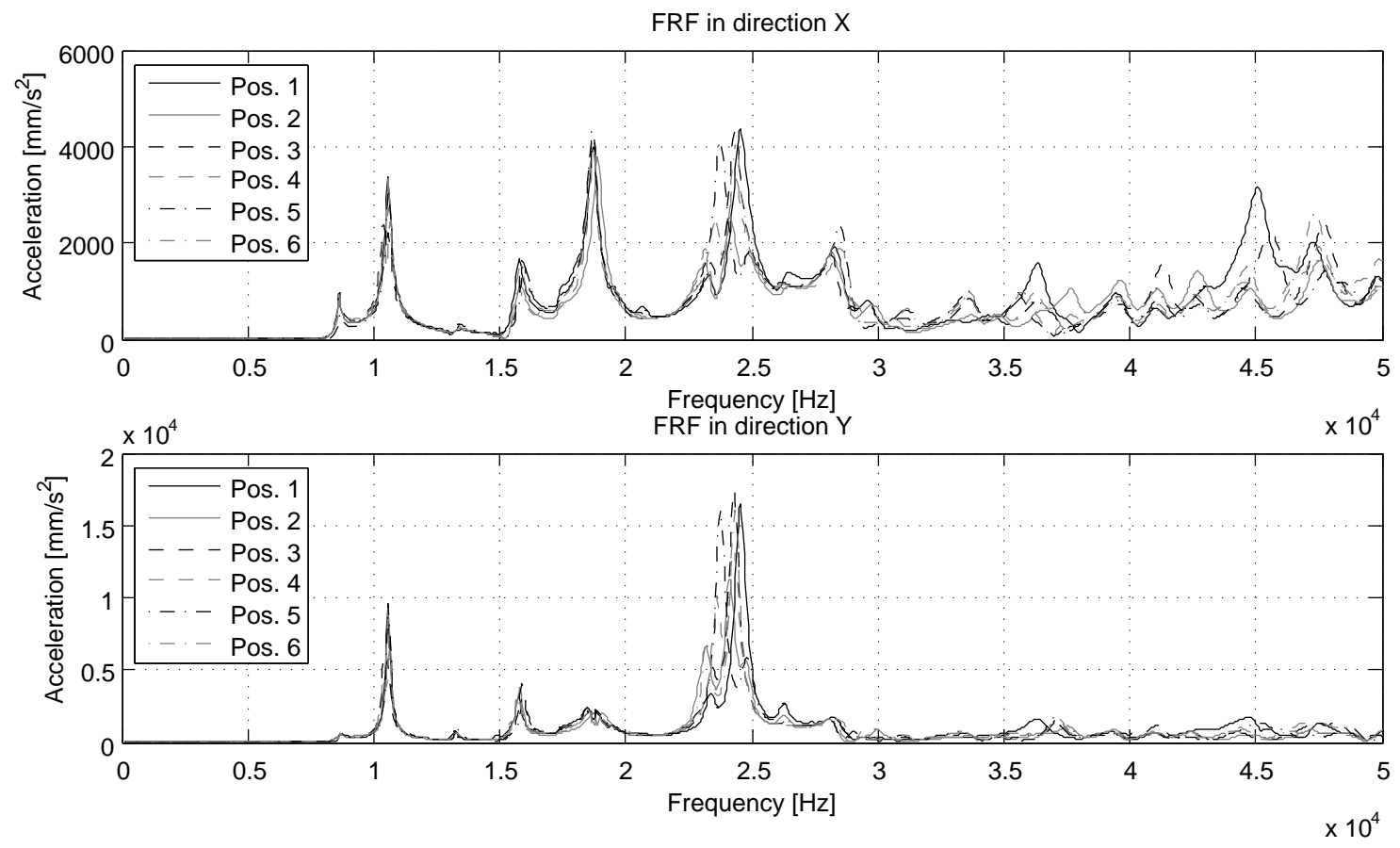

Figure 3: Frequency response functions of the high fidelity FE model. A single crack of $5 \mathrm{~mm}$ length is inserted in each of the stress concentration points.

the cracks in position 2 and 4 (see Figures $5 \mathrm{~b}$ and $5 \mathrm{~d}$ ). The reason for the non perfectly peaked posterior is two-fold: first, limited amount of reference data is available, and second, the RMSEs are already very close to zero (thus, within the target of the model updating procedure) for the model responses computed using crack length distributed according to the posterior PDF, as shown in Figure 6.

As a matter of fact, only two reference inertance FRFs are obtained from the high-fidelity FE model at a single location. Additional data, e.g., retrieved from a different point or with different loading condition, can improve the computation of the posterior at the expense of a more complex likelihood. It should be noted anyway that the approach carried out in this numerical example is a "worst case scenario" with regards to the availability of data, although the methodology was capable of giving a very good answer anyways.

\section{CONCLUSIONS}

A Bayesian updating procedure has been successfully employed as a computational framework to detect crack location and length. Reference dynamic data from vibration analysis has been used as target for the updating procedure.

The proposed framework allowed for successful updating and identification of crack position and length using simulated FE data. These preliminary results clearly show the possibility to apply this methodology to detect crack given data retrieved from sensors. To further validate this procedure, the updating procedure needs to be executed with additional numerical tests, e.g. with varying position and length of the crack. The results will show the performance of the framework, in particular with different crack length and location.

Future development and additional research will be taken by using real experimental data to further validate and expand the proposed approach, and using advanced more efficient Monte- 


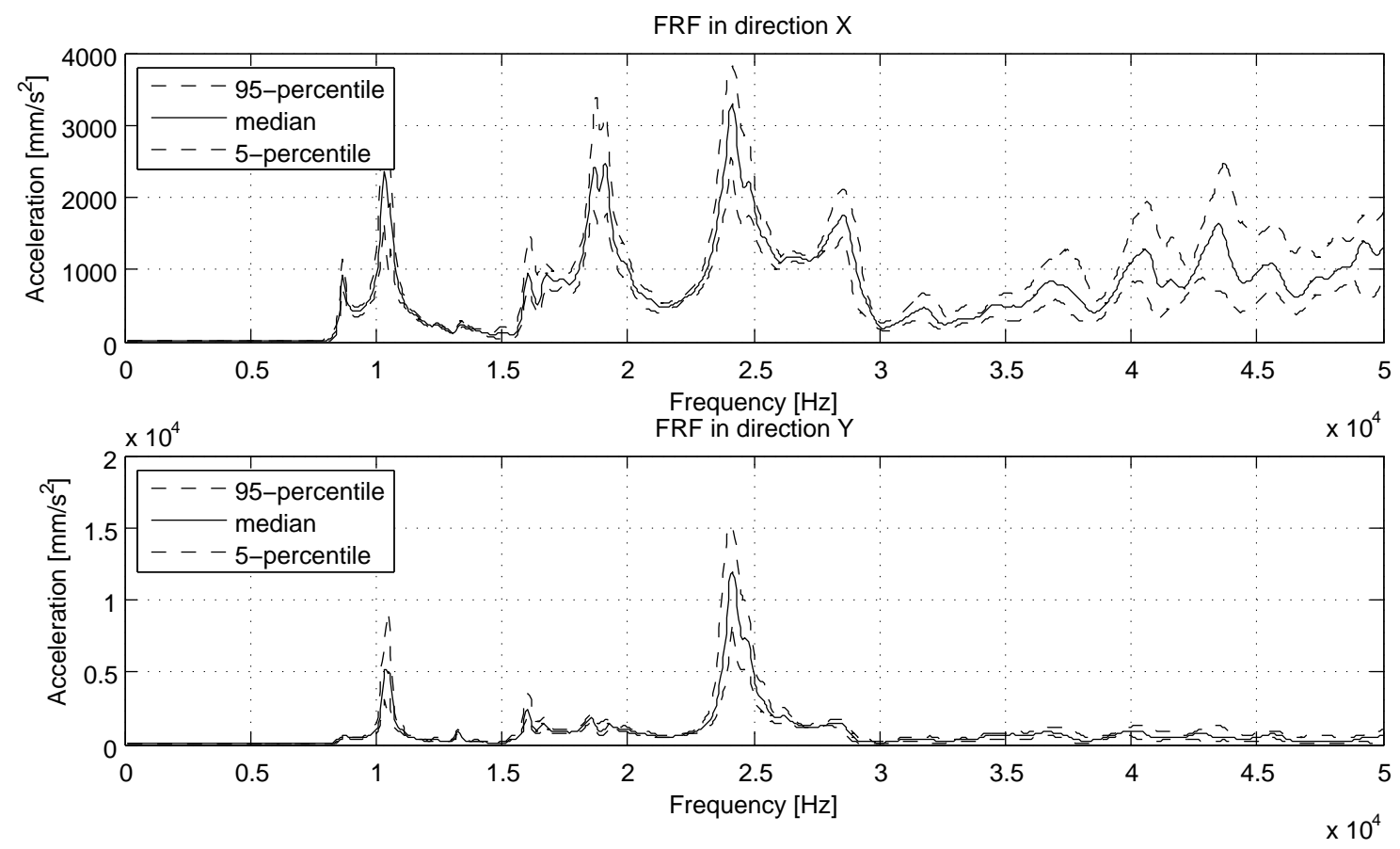

Figure 4: Variation of the FRFs with the crack length sampled from the prior distributions using the low-fidelity model. Random cracks of random length are inserted in all the possible positions.

Carlo procedures, i.e., sequential Monte-Carlo or Particle Filtering methods.

\section{Acknowledgement}

This work was undertaken at the Virtual Engineering Centre. The Virtual Engineering Centre is a University of Liverpool project partially funded by the Northwest Regional Development Agency and European Regional Development Fund.

\section{REFERENCES}

[1] P. Paris, F. Erdogan, A critical analysis of crack propagation laws. Transactions of the ASME. Series D, Journal of Basic Engineering, Volume 85, p. 52834, 1963.

[2] Beaurepaire, P., Valdebenito, M., Schuller, G., Jensen, H., 2012. Reliability-based optimization of maintenance scheduling of mechanical components under fatigue. Computer Methods in Applied Mechanics and Engineering, Volume 221-222, pp. 24-40.

[3] P. Chang, A. Flatau, S. Liu, Review paper: health monitoring of civil infrastructure. Structural Health Monitoring, Volume 2, 2003.

[4] C.-P. Fritzen, D. Jennewein, T. Kiefer, Damage detection based on model updating methods. Mechanical Systems and Signal Processing, 12(1), pp. 163-186, 1998.

[5] B. Goller, M. Broggi, A. Calvi, G.I. Schuëller, A stochastic model updating technique for complex aerospace structures. Finite Elements in Analysis and Design, 47(7), p. 739752, 2011. 


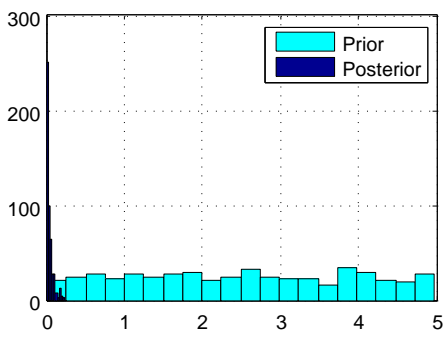

(a) Position 1

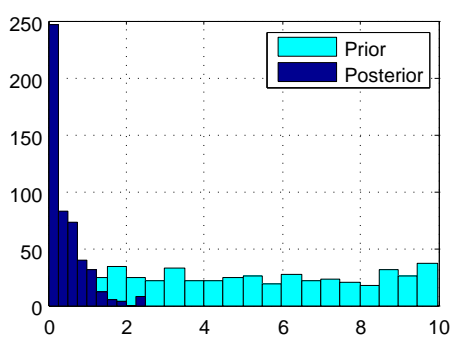

(d) Position 4

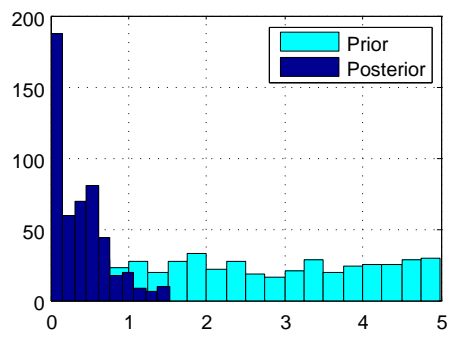

(b) Position 2

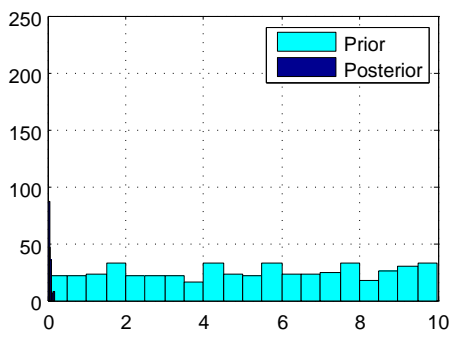

(e) Position 5

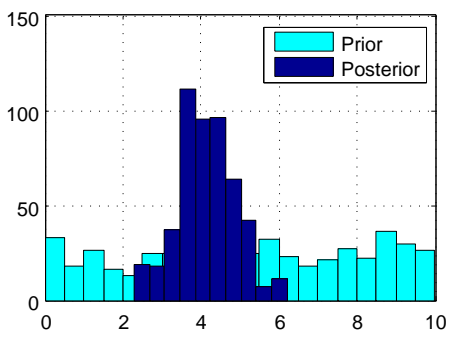

(c) Position 3

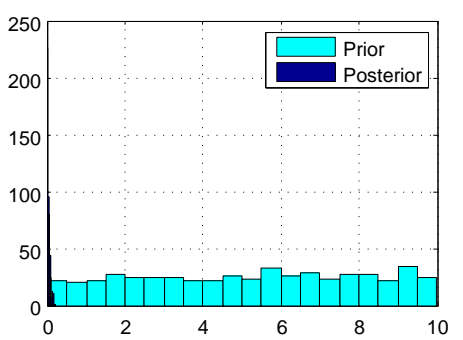

(f) Position 6

Figure 5: Prior (cyan) and Posterior (blue) distributions of the lengths of the cracks at the 6 candidate positions.

[6] E. Patelli, H.M. Panayirci, M. Broggi, B. Goller, P. Beaurepaire, H.M. Pradlwarter, G.I. Schuëller, General purpose software for efficient uncertainty management of large finite element models, Finite Elements in Analysis and Design, Volume 51, pp. 31-48, 2012.

[7] N. Moes, J. Dolbow, T. Belytschko, A finite element method for crack growth without remeshing, International Journal for Numerical Methods in Engineering, Volume 46, pp. 131-150, 1999.

[8] G. Zi, T. Belytschko, New crack-tip elements for XFEM and applications to cohesive cracks, International Journal for Numerical Methods in Engineering, Volume 57, pp. 2221-2240, 2003.

[9] T. Belytschko, T. Black, Elastic crack growth in finite elements with minimal remeshing. International Journal for Numerical Methods in Engineering, 45(5), pp. 601-620, 1999

[10] Y. Abdelaziz, A. Hamouine, A survey of the extended finite element. Computers \& Structures, 86(11-12), pp. 1141-1151, 2008

[11] S. Geniaut, Code Aster, notice d'utilisation de la methode X-FEM (in French). Technical Report U2.05.02, 2011.

[12] Bayes, T. An essay towards solving a problem in the doctrine of chances,. Philosophical Transactions of the Royal Society of London, Volume 53, pp. 370-418, 1763.

[13] J. L. Beck, L. S. Katafygiotis, Updating models and their uncertainties, I: Bayesian statistical framework. Journal of Engineering Mechanics, 124(4), p. 455, 1998. 


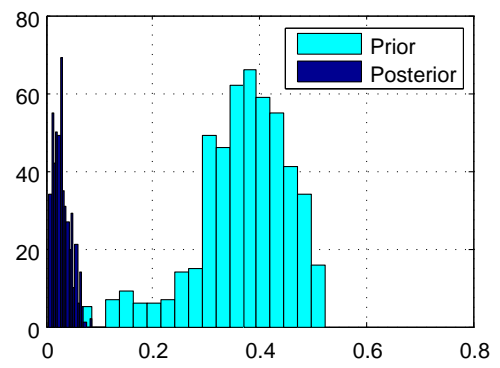

(a) $x$ direction

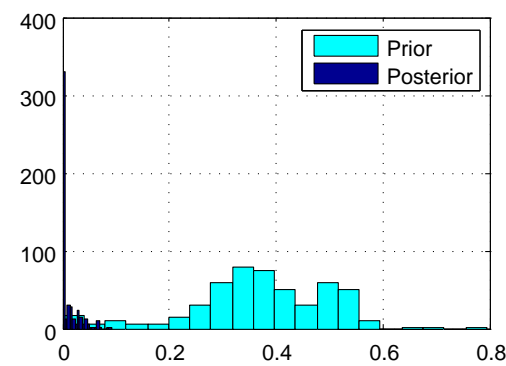

(b) $y$ direction

Figure 6: RMSE between the FRFs of the reference high-fidelity model with $5 \mathrm{~mm}$ crack in position 3 and of the low-fidelity model with random crack lengths in (a) $x$ and (b) $y$ directions. The cyan histogram denotes the random crack lengths of the low-fidelity model that have been extracted from the prior distribution, while in the blue histogram the crack lengths are distributed according to the posterior distribution.

[14] L. S. Katafygiotis, J. L. Beck, Updating models and their uncertainties. II: Model identifiability, Journal of Engineering Mechanics, 124(4), p. 463, 1998.

[15] J. Ching, Y.-C. Chen, Transitional Markov chain Monte Carlo method for Bayesian updating, model class selection, and model averaging, Journal of Engineering Mechanics, Volume 133, pp. 816-832, 2007.

[16] W. Hastings, Monte Carlo sampling methods using Markov chains and their applications. Biometrika, 57(1), pp. 97-109, 1970.

[17] M. Mrzyglod, A. Zielinski, Numerical implementation of multi-axial high-cycle fatigue criterion to structural optimization. Journal of Theoretical and Applied Mechanics, 44(3), pp. 691-712, 2006.

[18] Code_Aster, 2004. Code d'Analyse des Structures et Thermo-mcanique, pour des Etudes et des Recherches. [Online] Available at: http://www.code-aster.org/ [last access: 01-032013]. 